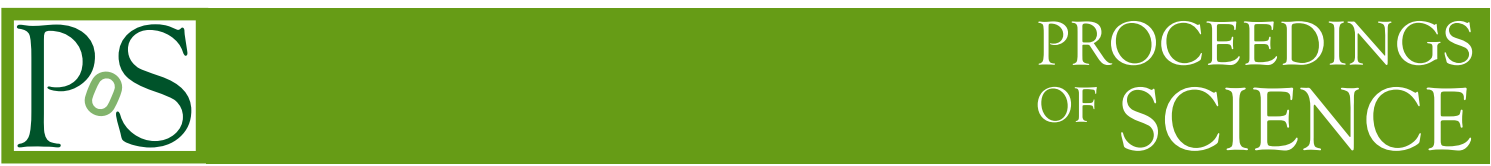

\title{
Electroweak Corrections at the LHC with MCFM
}

\section{John M. Campbell}

Fermilab, PO Box 500, Batavia, IL 60510, USA

E-mail: johnme@fnal.gov

\section{Doreen Wackeroth}

Department of Physics, SUNY at Buffalo, Buffalo, NY 14260, USA

E-mail: dow@ubpheno.physics.buffalo.edu

\section{Jia Zhou*}

Department of Physics, SUNY at Buffalo, Buffalo, NY 14260, USA

E-mail: jiazhou@buffalo.edu

Electroweak (EW) corrections at the LHC can be enhanced at high energies due to soft/collinear radiation of $W$ and $Z$ bosons, being dominated by Sudakov-like corrections in the form of $\alpha_{W}^{l} \log ^{n}\left(Q^{2} / M_{W}^{2}\right)\left(n \leq 2 l, \alpha_{W}=\frac{\alpha}{4 \pi \sin \theta_{W}^{2}}\right)$ when the energy scale $Q$ enters the TeV regime. Thus, the inclusion of EW corrections in LHC predictions is important for the search of possible signals of new physics in tails of kinematic distributions. EW corrections should also be taken into account in virtue of their comparable size $(\mathscr{O}(\alpha))$ to that of higher order QCD corrections $\left(\mathscr{O}\left(\alpha_{s}^{2}\right)\right)$. We calculated the next-to-leading-order (NLO) weak corrections to the neutral-current (NC) Drell-Yan process, top-quark pair production and di-jet producion, and implemented them in the Monte-Carlo program MCFM. This enables a combined study with the corresponding NLO QCD corrections. We provide both the full NLO weak corrections and their weak Sudakov approximation valid at high energies. The latter is often used for a fast evaluation of weak effects, and having the exact result available as well allows to quantify the validity of the Sudakov approximation.

XXIII International Workshop on Deep-Inelastic Scattering 27 April - May 12015

Dallas, Texas

\footnotetext{
*Speaker.
} 


\section{Introduction}

As the LHC reaches an unprecedented high energy and high precision, the inclusion of electroweak (EW) corrections becomes increasingly important in testing the Standard Model (SM) and searching for signals of new physics, in particular in the high-energy and high-momentum regimes of kinematic distributions. Electroweak corrections may also play a significant role in the extraction of parton distribution functions (PDF), for instance in constraining the gluon PDF at high momentum fraction in di-jet production. However, the calculations of EW corrections to relevant processes are often not readily available in public codes and can quickly become complicated (and CPU intensive) for high multiplicities. Even with the increasing availability of automated tools for the calculation of EW corrections, a dedicated and efficient computation for specific processes which are treated at the same footing as QCD corrections in a widely used Monte Carlo program such as MCFM [1] is still highly desirable for LHC studies. As a first step to improve predictions for the LHC at high energies, one could implement the Sudakov approximation of EW corrections (see, e.g., [2] for a review). One example of such an application is the weak Sudakov corrections to $Z+\leq 3$ jets implemented in ALPGEN [3]. Our goal is to implement weak corrections in MCFM, so that they become readily available to the experimental community and can be studied together with the already implemented QCD corrections. So far, we implemented the weak 1-loop corrections to the neutral-current (NC) Drell-Yan process, $p p \rightarrow \gamma, Z \rightarrow l^{+} l^{-}$, top-quark pair production, and di-jet production. The implementation of these processes in MCFM includes both the Sudakov approximation for weak corrections valid at high energies and the complete 1-loop weak corrections to be able to quantify the goodness of the Sudakov approximation. Earlier calculations and studies of weak 1-loop corrections to these processes can be found in Refs. [4, 5, 6], which we used to cross-check our calculation and implementation. The general algorithm of Denner-Pozzorini [7] is adopted in the implementation of the weak Sudakov approximation in MCFM. Here we present preliminary results of the implementation of weak corrections in MCFM for the processes under study for a number of relevant kinematic distributions. In particular, in Sections 3, 4, and 5 we compare the results of the Sudakov approximation with the ones of the complete EW 1-loop calculation for NC Drell-Yan, top-pair and di-jet production, respectively.

\section{Implementation of Weak Corrections in MCFM}

We investigate the SM weak 1-loop corrections to the processes discussed in Sect. 3, 4, and 5, i.e., the $\mathscr{O}(\alpha)$ corrections that include the virtual contribution of massive gauge $V^{a}\left(Z / W^{ \pm}\right)$and Higgs bosons in higher order diagrams. Figure 1 shows sample Feynman diagrams that contribute to these relative (virtual) corrections in NC Drell-Yan, top-quark pair and di-jet production. In the latter two processes, however, we need to take into account also NLO QCD corrections to the interference of the QCD-EW mixed leading-order (LO) contribution to achieve the same order of $\mathscr{O}\left(\alpha \alpha_{s}^{2}\right)$, which are illustrated in Fig.1 (b.2) and (c.2). These NLO QCD virtual corrections require the inclusion of the real correction with emission/absorption of a gluon to cancel the IR divergence. On the other hand, photonic corrections are not included since they themselves form a separate gauge invariant subset and are not enhanced at high-energy scales. 


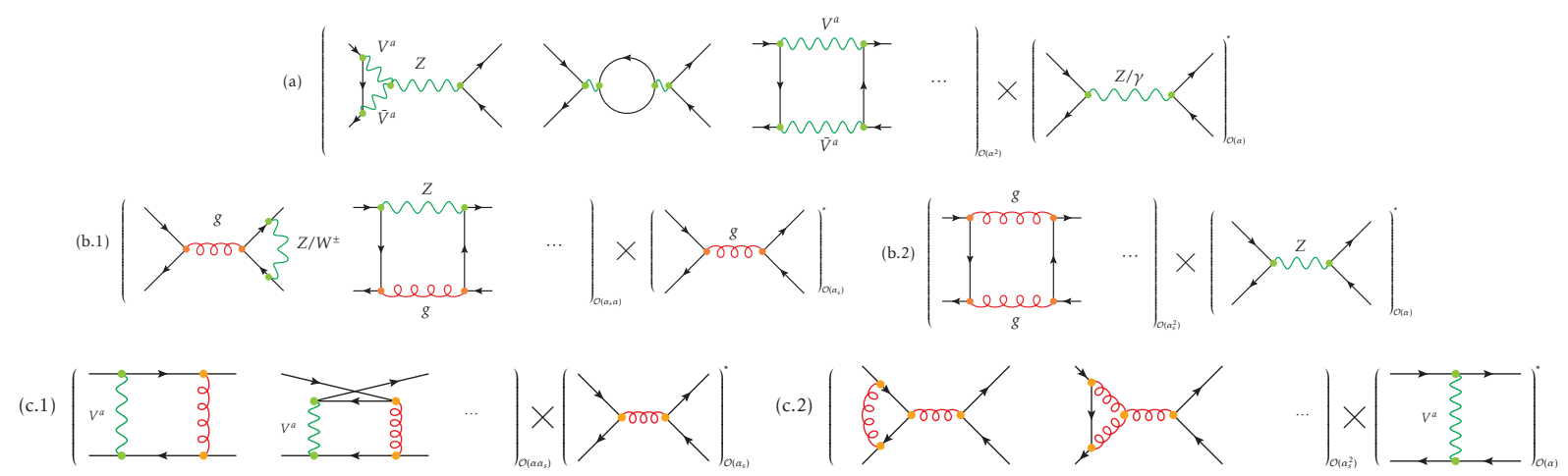

Figure 1: Sample Feynman diagrams for the $\mathscr{O}(\alpha)$ weak corrections to the LO cross sections of the NC Drell-Yan process (a), top-quark pair production (b.1), and di-jet production (c.1). Figures (b.2) and (c.2) illustrate $\mathscr{O}\left(\alpha_{s}\right)$ QCD corrections to the interference of QCD-EW mixed LO cross sections which arise in the case of four-quark external states in top-quark pair and di-jet production.

We use the on-shell renormalization scheme and a constant gauge boson decay width in the $Z / W^{ \pm}$ propagators in the calculation. To produce the numerical results we use the following input parameters:

$$
\begin{aligned}
& G_{\mu}=1.16639 \times 10^{-5} \mathrm{GeV}^{-2}, \alpha_{\mu}=1 / 132.5605045, \sin ^{2} \theta_{W}=1-M_{W}^{2} / M_{Z}^{2} \\
& M_{Z}=91.1876 \mathrm{GeV}, \Gamma_{Z}=2.4952 \mathrm{GeV}, M_{W}=84.425 \mathrm{GeV}, \Gamma_{W}=2.141 \mathrm{GeV} \\
& M_{H}=120 \mathrm{GeV}, m_{t}=173.2 \mathrm{GeV}, m_{b}=4.6 \mathrm{GeV}, m_{u}=66 \mathrm{MeV}, m_{d}=66 \mathrm{MeV} \\
& m_{c}=1.2 \mathrm{GeV}, m_{s}=150 \mathrm{MeV}, m_{e}=0.51099892 \mathrm{MeV}, m_{\mu}=105.658369 \mathrm{MeV}, \\
& m_{\tau}=1.777 \mathrm{GeV}
\end{aligned}
$$

Note that the quarks and leptons except for the top quark are treated massless if those particles are initial/final-state particles. We only retain their masses in closed fermion loops, i.e. when calculating the gauge boson self-energy corrections in the NC Drell-Yan process. The factorization scale $\mu_{F}$ is set to be equal to the renormalization scale $\mu_{R}$, and is chosen to be the mass of the $Z$ boson $M_{Z}$, twice of the mass of the top quark $2 m_{t}$, and the transverse momentum of the leading jet $p_{T, j_{1}}$, in NC Drell-Yan, top-pair and di-jet production, respectively.

\section{Neutral Current Drell-Yan Process}

The partonic level process under consideration is $q \bar{q} \rightarrow l \bar{l}$ with exchange of a neutral EW gauge boson $(\gamma / Z)$, where $q \in\{u, d, c, s, b\}$ denotes initial-state quarks, and $l \in\left\{e, \mu, \tau ; v_{e}, v_{\mu}, v_{\tau}\right\}$ finalstate leptons. The LO cross section is thus of $\mathscr{O}\left(\alpha^{2}\right)$. When we consider NLO weak corrections to the LO process, it refers to a weak gauge boson exchange in vertex, self-energy, and box corrections as demonstrated in Fig.1 (a). In order to better understand and characterize the validity of the Sudakov approximation, we produce differential distributions of relative corrections with respect to the invariant mass $M_{l^{+} l^{-}}$and transverse momentum $p_{T, l^{+}\left(l^{-}\right)}$using the exact 1-loop calculation 
and Sudakov approximation. The relative correction $\delta$ is defined as follows,

$$
\delta=\frac{\delta \sigma^{1-l o o p}}{\sigma_{L O}}=\frac{\sigma_{N L O}-\sigma_{L O}}{\sigma_{L O}},
$$

where $\sigma_{L O}$ and $\sigma_{N L O}$ are the LO and NLO cross sections.

Figure 2 shows $\delta$ for both distributions for $p p \rightarrow \gamma, Z \rightarrow l^{+} l^{-}$at the LHC at $\sqrt{s}=14 \mathrm{TeV}$. The results are produced with the PDF set MRST2004QED [8]. As can be seen the Sudakov approximation shows good agreement with the exact NLO calculation in both distributions, and the largest discrepancy which appears in the invariant mass distribution is as small as a few percent $(\sim 2 \%-3 \%)$ at $8 \mathrm{TeV}$.
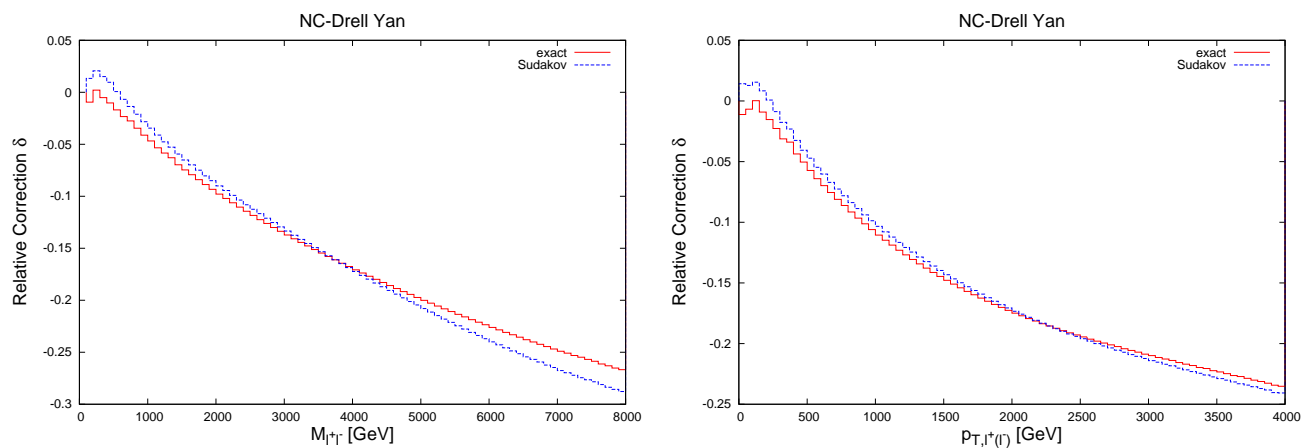

Figure 2: The relative correction $\delta$ to the invariant mass $M_{l^{+} l^{-}}$(left) and the transverse momentum $p_{T, l^{+}\left(l^{-}\right)}$ (right) distributions at the LHC for $\sqrt{s}=14 \mathrm{TeV}$, for the NC Drell-Yan process $p p \rightarrow l^{+} l^{-}$. In each plot, the red curve denotes the distribution obtained with the exact NLO calculation, while the blue one denotes the one obtained with the Sudakov approximation. No cuts have been applied.

\section{Top-Quark Pair Production}

At the LHC, top-quark pairs are dominantly produced via strong quark-antiquark annihilation and gluon fusion at the parton level, with an LO cross section of $\mathscr{O}\left(\alpha_{s}^{2}\right)$. We consider the 1-loop weak contribution of $\mathscr{O}\left(\alpha \alpha_{s}^{2}\right)$ to these top-quark pair production processes. In addition to the weak 1-loop corrections that are present in both channels, the quark-antiquark annihilation channel includes the QCD corrections to the interference of QCD-weak mixed LO contributions. These are comprised of the virtual box corrections shown in Fig.1 (b.2), as well as the corresponding real corrections with emission/absorption of a gluon. We use the PDF set CTEQ6L [9] to produce the numerical results in Fig.3 and Fig. 4.

Figure 3 shows the results for the relative corrections at the LHC at $\sqrt{s}=14 \mathrm{TeV}$, where the upper(lower) two plots show the results for the invariant mass $M_{t \bar{t}}$ and transverse momentum $p_{T, t(\bar{t})}$ distributions in the quark-antiquark annihilation(gluon fusion) channel. As expected the Sudakov approximation works better in case of quark-antiquark annihilation, since this subprocess is analogous to the NC Drell-Yan at high energies where the effect of the mass of the top quark is negligible. In contrast, in the gluon fusion channel, there is an obvious discrepancy between the Sudakov approximation and the exact NLO calculation in the $t \bar{t}$ invariant mass distribution while in the topquark transverse momentum distribution, it is not as significant. After further investigation, it is 

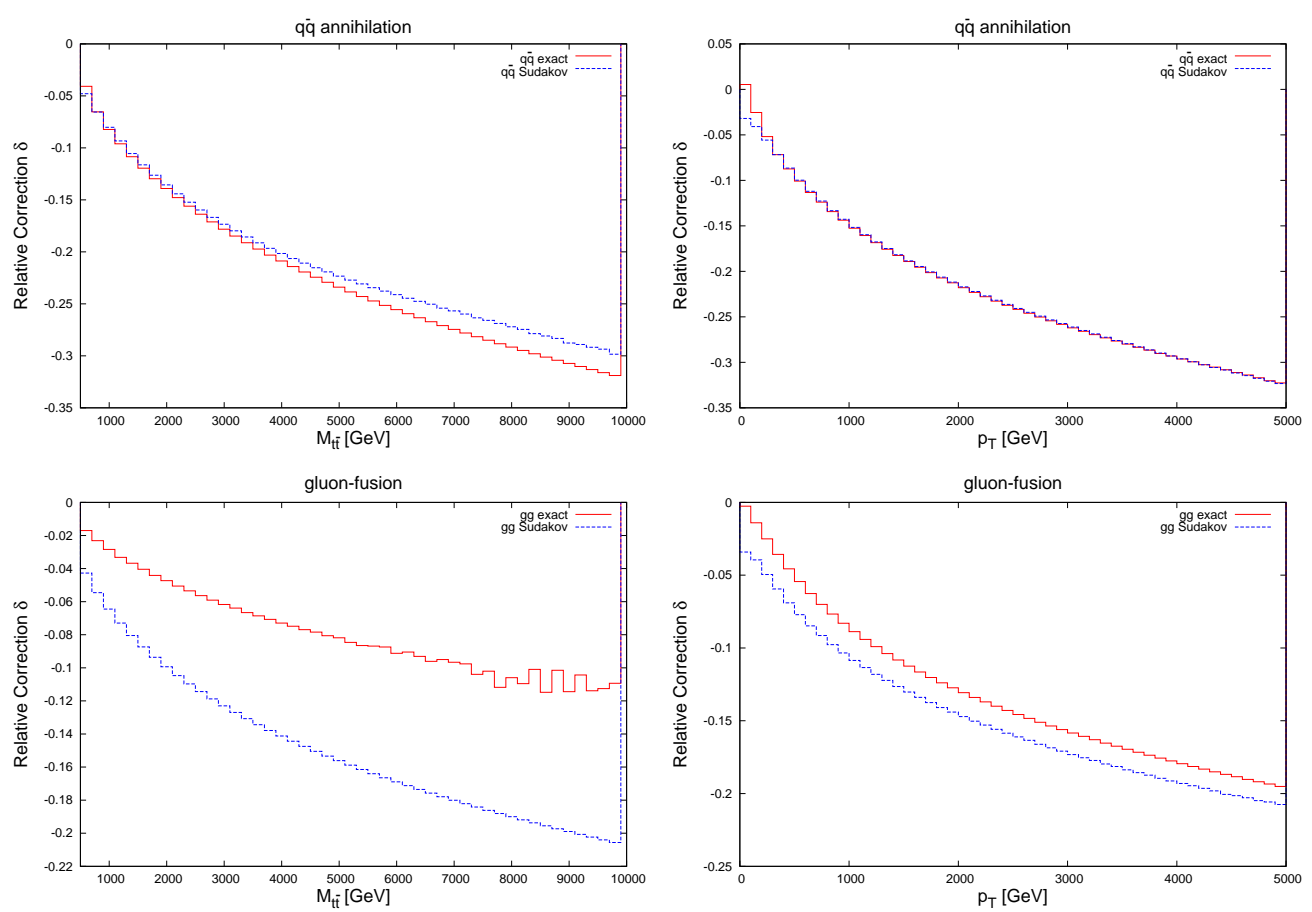

Figure 3: The relative correction $\delta$ to the invariant mass $M_{t \bar{t}}$ and transverse momentum $p_{T, t(\bar{t})}$ distributions in top-quark pair production at the LHC at $\sqrt{s}=14 \mathrm{TeV}$. No cuts have been applied. The upper and lower two plots show respectively the results for the quark-antiquark annihilation and gluon fusion channel. Again the red curves denote the exact NLO calculation and the blue curves the Sudakov approximation.

clear that this disagreement is caused by the mismatch of the angular dependence because there is no such information captured by the Sudakov approximation in the gluon fusion channel. Since the transverse momentum distribution has less dependence on the scattering angle, or equivalently rapidity, it is expected that the Sudakov approximations works better in this case. The invariant $t \bar{t}$ mass is a function of rapidity, $M_{t \bar{t}}^{2}=2 m_{t}^{2}+2 m_{T}^{2} \cosh \left(y_{t}-y_{\bar{t}}\right)+2 p_{T}^{2}$, where $m_{T}=\sqrt{p_{T}^{2}+m_{t}^{2}}$, and $y_{t(\bar{t})}$ denotes rapidity. We therefore expect to find a better agreement between the Sudakov approximation and the exact result by imposing a cut in the top-quark rapidity. This is illustrated in Fig.4 where we show results for different rapidity cuts. We indeed find agreement when $\left|y_{t, \bar{t}}\right|<1$. This constrains the range of validity of the Sudakov approximation, as has been also pointed out in Ref. [10].

\section{Di-jet Production}

Di-jet production is described at LO by $2 \rightarrow 2$ processes involving quarks and gluons, where the two final-state partons are detected as jets after hadronization. We thus categorize the processes contributing to di-jet production into three subprocesses according to the number of external quarks or gluons, which are four-quark, two-gluon-two-quark, and four-gluon subprocesses. At tree level, these processes can be produced via strong or EW interactions. Thus, the LO cross section in di-jet production consists of the purely QCD contributions of $\mathscr{O}\left(\alpha_{s}^{2}\right)$, the mixed QCD-EW contribution 


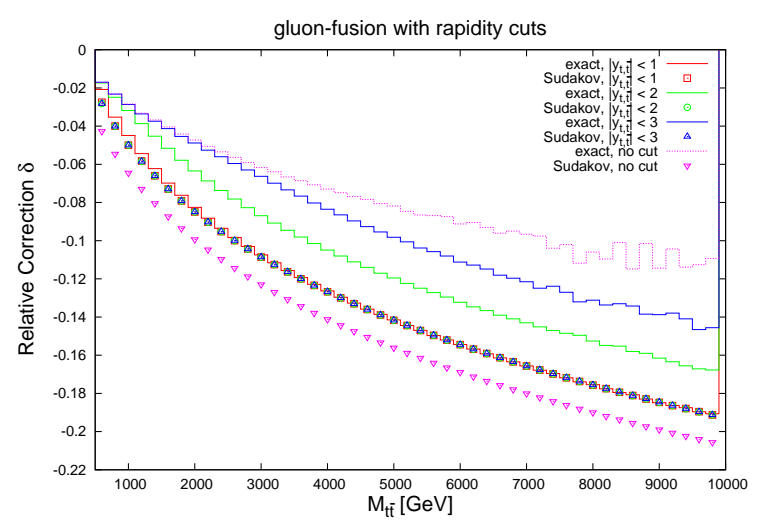

Figure 4: The relative correction $\delta$ to the $M_{t \bar{t}}$ distribution in top-quark pair production at the LHC at $\sqrt{s}=14 \mathrm{TeV}$ for different cuts on $\left|y_{t, \bar{t}}\right|$. The results represented by symbols are those of the Sudakov approximation and the lines indicate the results obtained with the exact NLO calculation.

of $\mathscr{O}\left(\alpha_{s} \alpha\right)$, as well as the purely EW contribution of $\mathscr{O}\left(\alpha^{2}\right)$. The NLO corrections consist of virtual and real corrections, where the latter exist in the four-quark subprocesses associated with a emitted/absorbed gluon. And there are two types of the interference of diagrams that contribute to the full NLO corrections of $\mathscr{O}\left(\alpha \alpha_{s}^{2}\right)$, and symbolically, we can write them as

$$
\hat{\sigma}\left(\alpha \alpha_{s}^{2}\right) \propto\left\{\begin{array}{l}
2 \operatorname{Re}\left[\mathscr{M}\left(\alpha_{s} \alpha\right) \cdot \mathscr{M}^{*}\left(\alpha_{s}\right)\right] \\
2 \operatorname{Re}\left[\mathscr{M}\left(\alpha_{s}^{2}\right) \cdot \mathscr{M}^{*}(\alpha)\right]
\end{array}\right.
$$

where $\mathscr{M}\left(\alpha_{s}\right)$ and $\mathscr{M}(\alpha)$ denote the LO amplitude with gluon and weak boson exchange, respectively, and $\mathscr{M}\left(\alpha_{s}^{2}\right)$ the NLO QCD correction to the strong LO amplitude. In the virtual corrections, $\mathscr{M}\left(\alpha_{s} \alpha\right)$ denotes the NLO amplitude that can be either weak correction to the strong LO amplitude, or QCD correction to the weak LO amplitude; while in the real correction, the NLO amplitude $\mathscr{M}\left(\alpha_{s} \alpha\right)$ is restricted to the latter because we only need gluon radiation in the real contribution to cancel the IR divergence in the virtual contribution. Note that there is no NLO weak correction to the four-gluon subprocesses.

We calculate the inclusive jet process using the anti- $k_{T}$ jet clustering algorithm [11], and set the pseudo-cone size to $R=0.6$. We apply the following cuts on the jet transverse momentum and jet rapidity:

$$
p_{T, j}>25 \mathrm{GeV}, \quad\left|y_{j}\right|<2.5 \text {. }
$$

We use the PDF set CTEQ6L1 [9]. Figure 5 shows preliminary results for the relative correction at the LHC at $\sqrt{s}=14 \mathrm{TeV}$. The comparison between the Sudakov approximation and exact NLO correction is restricted to the one-loop weak relative correction of $\mathscr{O}(\alpha)$, i.e., the LO cross section in Eq.(3.1) is purely QCD, and the total NLO result does not contain the LO EW effect. We find that Sudakov approximation gives less negative contribution than the complete set of NLO corrections that amount to the contribution of $\mathscr{O}\left(\alpha \alpha_{s}^{2}\right)$. There might be two reasons for this disagreement between the approximation and the exact calculation. One is the missing angular dependence in the Sudakov approximation in the two-gluon-two-quark subprocesses, which is similar to what we observed in the gluon fusion channel in top-quark pair production. The second is that we neglected the QCD corrections to the EW LO amplitude in the Sudakov approximation. Therefore, we may 
only be able to use the Sudakov approximation in some particular subprocesses such as four-quark processes, and a cut on the scattering angle (or rapidity) should be imposed when the two-gluontwo-quark subprocesses are included. Final results and a more detailed discussion will be made available in Ref. [12].

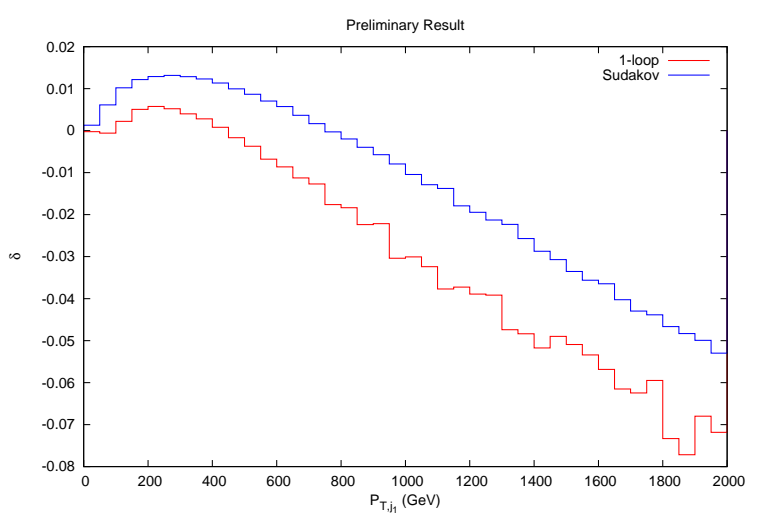

Figure 5: The relative correction $\delta$ to the transverse momentum distribution of the leading jet $p_{T, j_{1}}$ in di-jet production at the LHC at $\sqrt{s}=14 \mathrm{TeV}$. Again the red curve denotes the exact NLO calculation and the blue curve the Sudakov approximation.

\section{Acknowledgments}

This research is supported in part by the US DOE under contract DE-AC02-07CH11359 and the NSF under award no. PHY-1118138.

\section{References}

[1] J. M. Campbell and R. K. Ellis, Nucl. Phys. Proc. Suppl. 205-206, 10 (2010).

[2] J. Butterworth, G. Dissertori, S. Dittmaier, D. de Florian, N. Glover, K. Hamilton, J. Huston and M. Kado et al., arXiv:1405.1067 [hep-ph].

[3] M. Chiesa et al, Phys. Rev. Lett. 111 (2013) 121801.

[4] U. Baur, O. Brein, W. Hollik, C Schappacher, and D. Wackeroth, Phys. Rev. D 65 (2002) 033007.

[5] W. Beenakker et al, Nucl. Phys. B 411(1994) 343; J. H. Kühn, A. Scharf and P. Uwer, Eur. Phys. J. C 45 (2006) 139; J. H. Kühn, A. Scharf and P. Uwer, Eur. Phys. J. C 51 (2007) 37.

[6] S. Dittmaier, A. Huss and C. Speckner, JHEP 1211 (2012) 095; A. Scharf, arXiv:0910.0223 [hep-ph].

[7] A. Denner and S. Pozzorini, Eur. Phys. J. C 18 (2001) 461; A. Denner and S. Pozzorini, Eur. Phys. J. C 21 (2001) 63; A. Denner, B. Jantzen, and S. Pozzorini, JHEP 0811 (2008) 062.

[8] A. D. Martin, R. G. Roberts, W. J. Stirling,and R. S. Thorne, Eur. Phys. J. C 39 (2005) 155.

[9] J. Pumplin, D. R. Stump, J. Huston, H. L. Lai, P. Nadolsky, and W. K. Tung, JHEP 0207 (2002) 012.

[10] S. Moretti, M. R. Nolten, and D. A. Ross, Phys. Lett. B 639 (2006) 513; J. H. Kühn, A.Scharf, and P.Uwer, Phys. Rev. D 91 (2015) 1, 014020.

[11] M. Cacciari, G. P. Salam, and G. Soyez, JHEP 0804 (2008) 063.

[12] J. Campbell, D. Wackeroth, and J. Zhou, in preparation. 